\title{
Investigation of galectin-3, lipocalin 2, retinol binding protein (RBP), small dense low-density lipoprotein (sdLDL) in patients with hirsutism
}

\author{
Ibrahim Halil Yavuz¹, Goknur Ozaydin-Yavuz¹, Erdem Çokluk², Zehra Kurtoğlu³, Serap Gunes Bilgili
}

'Department of Dermatology, University Faculty of Medicine, Van Yuzuncu Yil, Van, Turkey 2Department of Biochemistry, Faculty of Medicine, Yuzuncu Yil University, Van, Turkey

${ }^{3}$ Department of Obstetrics and Gynecology, Ankara Research and Training Hospital, Ankara, Turkey

Adv Dermatol Allergol 2019; XXXVI (2): 177-183

DOI: https://doi.org/10.5114/ada.2019.84593

\begin{abstract}
Introduction: Hirsutism is defined as excessive terminal hair in androgen-dependent areas in women. Adipose tissue is no longer regarded as a storage site for triglycerides or as a source of free fatty acids but is currently emerging as a key constituent of energy metabolism secreting numerous enzymes, cytokines, growth factors, and hormones.

Aim: To evaluate serum levels of galectin-3 (Gal3), lipocalin-2 (LCN2), retinol binding protein (RBP), and small dense low-density lipoprotein (sdLDL) in patients with hirsutism and patients co-presenting with polycystic ovary syndrome (PCOS) and hirsutism.

Material and methods: The study included 90 patients that were divided into three groups: (I) patients with idiopathic hirsutism (IH) $(n=30)$, (II) PCOS patients with hirsutism (H-PCOS) $(n=30)$, and (III) the control group $(n=30)$. Informed consent was obtained from each participant. Idiopathic hirsutism was scored using the modified Ferriman-Gallwey ( $\mathrm{mFG}$ ) scoring system and the diagnosis of PCOS was established based on the modified Rotterdam criteria. Human Gal3, LCN2, RBP, and sdLDL levels were analysed using a commercially available double-antigen sandwich enzyme-linked immunosorbent assay.

Results: No significant difference was found between the three groups with regard to serum Gal3, LCN2, RBP, and sdLDL levels $(p>0.05)$. Median values were higher in the H-PCOS group than in the other two groups: body weight (median: $76.5 \mathrm{~kg})(p<0.001)$, waist circumference (median: $82.5 \mathrm{~cm})(p=0.001)$.

Conclusions: No significant correlation was found between the adipokines that play a role in the aetiology of numerous diseases and some mediators of the lipid metabolism and hirsutism.
\end{abstract}

Key words: hirsutism, polycystic ovary syndrome, adipokines.

\section{Introduction}

Hirsutism is defined as excessive terminal hair in androgen-dependent areas in women. Hirsutism is more than just a cosmetic problem for women because it has negative effects on their self-image and quality of life [1].

Hirsutism can result from androgenic or non-androgenic factors, or may be idiopathic. However, it mostly results from androgenic factors, with the most common cause being polycystic ovary syndrome (PCOS). Patients with no apparent cause of hirsutism are accepted to have idiopathic hirsutism $(\mathrm{IH})$ [2]. The exact pathogenesis of $\mathrm{IH}$ remains unknown. Hirsutism is considered to be caused by an inter- action between androgen levels and the sensitivity of the hair follicle to androgens [2, 3]. Previous studies indicate that patients with $\mathrm{IH}$ have normal serum androgen levels. However, increased local $5 \alpha$-reductase activity in the skin is considered as the hallmark of hirsutism [3].

Polycystic ovary syndrome, which was first defined by Stein and Leventhal in 1935, is an endocrine disorder affecting 5-20\% of women of reproductive age. Although the exact aetiology of PCOS remains unclear, several genetic and environmental factors have been observed in numerous studies [4, 5]. The PCOS is mostly characterized by hyperandrogenism and chronic anovulation. However, the diagnostic criteria for PCOS remain controver-

Address for correspondence: Dr. Ibrahim Halil Yavuz, Department of Dermatology, Faculty of Medicine, Yuzuncu Yil University, 65100 Van, Turkey, phone: +90 05054753361, e-mail: ihalilyavuz@gmail.com Received: 27.12.2017, accepted: 12.01 .2018$. 
sial. The PCOS has four main phenotypes: classic type 1 , classic type 2, ovulatory, and normoandrogenic [6, 7].

Adipose tissue is no longer regarded as a storage site for triglycerides or as a source of free fatty acid but is currently emerging as a key constituent of energy metabolism secreting numerous enzymes, cytokines, growth factors, and hormones [8]. Moreover, the mature adipocytes available in the adipose tissue are accepted as an active endocrine organ secreting numerous mediators that participate in various metabolic reactions [9]. These active mediators are secreted from white adipose tissue and are termed 'adipokines'. Adipokines play a role in numerous physiological processes including nutrition, appetite, energy expenditure, insulin and glucose metabolisms, lipid metabolism, regulation of blood pressure, vascular remodelling, coagulation, and inflammation [10].

Galectin-3 (Gal3) is a member of the lectin family and has a molecular weight of about $30 \mathrm{kDa}$. Like all galectins, gal 3 also includes amino acids that bind to $\beta$-galactosides [11]. Gal3 is responsible for a number of processes including apoptosis, cell cycle, cell growth, cell activation, and cell adhesion. Moreover, it has been shown to have a major role in heart failure, cancer, inflammation, fibrosis, stroke, diabetes, obesity, and atherosclerosis. In addition, it has been reported to play a key role in impaired follicular growth in patients with PCOS [12, 13].

Retinol-binding protein (RBP) is known as the primary carrier protein that binds to retinol in the plasma. RBP is currently emerging as a potential adipokine that modulates insulin resistance and glucose metabolism. Moreover, RBP has been shown to be associated with insulin resistance and to be elevated in patients with PCOS [14-16].

Lipocalin-2 (LCN2), also known as neutrophil gelatinase-associated lipocalin (NGAL), is a lipophilic lipoprotein of the lipocalin superfamily. LNC2 is associated with numerous physiological processes such as inflammation, apoptosis, transport of retinal and retinoic acid, cholesterol, and transport of prostaglandins. LCN2 is considered to be secreted from adipocytes, neutrophils, uterus endothelial cells, and the cells in the brain, colon, and lungs. Moreover, LCN2 has been shown to be elevated in several diseases including atherosclerosis, diabetes type 2, and PCOS [17-19].

Small, dense low-density lipoprotein (sdLDL) is more atherogenic than the other lipoproteins and has been shown to be strongly associated with coronary artery disease [20].

In this study, we aimed to evaluate serum levels of Gal3, LCN2, RBP, and sdLDL in patients with hirsutism and patients co-presenting with PCOS and hirsutism.

\section{Material and methods}

The study included 90 patients who presented to the Yuzuncu Yil University Medical School Dermatology Department between 2015 and 2017. The patients were divided into three groups: (I) patients with idiopathic hirsutism $(\mathrm{IH})(n=30)$, (II) PCOS patients with hirsutism (H-PCOS) $(n=30)$, and (III) the control group $(n=30)$. Informed consent was obtained from each participant. An approval was obtained from local ethics committee with approval number 90 dated 2 June 2015. The study protocol was prepared in accordance with the Declaration of Helsinki. Patient characteristics including age, gender, body height and weight, menstrual status, waist circumference (WC), diastolic blood pressure, body mass index (BMI), and status of smoking were recorded. Hormone profile testing was performed on days 3-7 of the menstrual cycle.

In the IH group, idiopathic hirsutism was scored using the modified Ferriman-Gallwey (mFG) scoring system. With this system, nine body areas including the upper lip, chin, chest, upper back, lower back, upper abdomen, lower abdomen, arm, and thigh were evaluated separately. Each area was scored between 0 and 4, where 0 indicates no presence of hair and 4 indicates presence of excessive hair. Hirsutism was represented by a score of 8 or above. Patients with a history of the irregular menstrual cycle and a known diagnosis of hirsutism, patients with an ongoing pregnancy, hyperprolactinemia, Cushing's syndrome, thyroid disorder and hepatic disease, and those who were breastfeeding and using hormone drugs were excluded from the $\mathrm{IH}$ group.

In the H-PCOS group, the patients were selected based on the modified Rotterdam criteria [21]. The diagnosis of PCOS was based on the presence of two of the three criteria: (I) oligo- or anovulation, (II) clinical and/ or biochemical signs of hyperandrogenism, (III) ultrasonographic signs of polycystic ovaries and the exclusion of other disorders (e.g. Cushing's syndrome, congenital adrenal hyperplasia, and androgen secreting tumours). The patients were included into this group if they had an mFG score of 8 or above.

The control group included 30 women that presented to the Dermatology Department for routine examination of various diseases such as contact dermatitis, tinea pedis, and itching and had a history of regular menstrual cycles. All the patients had a normal hormone profile and had no other known disease.

Anthropometric measurements included weight (kg), height $(\mathrm{cm})$, and waist circumference $(\mathrm{cm})$. Body mass index was calculated by the weight in kilograms divided by the square of the height. The venous blood samples obtained during the routine dermatological examinations were collected into non-anticoagulant yellow-top tubes and were transferred for biochemical analysis. Following the coagulation process, the samples were centrifuged at 1,500 g/rpm for $10 \mathrm{~min}$ and the serum was separated. The samples were then divided into sections and stored at $-40^{\circ} \mathrm{C}$ until analysis. Human Gal3, LCN2, RBP, and sdLDL levels were analysed using a commercially available double-antigen sandwich enzyme-linked immuno- 
sorbent assay (ELISA) (Eastbiopharm ${ }^{\circledR}$, Zhejiang, China). The results were expressed as Gal3 (pg/ml), LCN2 (ng/l), $\operatorname{RBP}(\mathrm{ng} / \mathrm{ml})$, and sdLDL $(\mathrm{mmol} / \mathrm{l})$.

\section{Statistical analysis}

Data were analysed using SPSS 17.0 for Windows (SPSS Inc., Chicago, IL, USA). Descriptive statistics were expressed as mean, standard deviation (SD), median, interquartile range (IQR), minimum, and maximum. The Pearson correlation coefficient, $\chi^{2}$ test, and Fisher's exact test were used for comparing $2 \times 2$ tables. Parametric variables with normal distribution were compared by using independent $t$-test for two groups and one-way ANOVA for three or more groups. Nonparametric variables with non-normal distribution were compared by using Mann Whitney $U$ test for two groups and Kruskal-Wallis test for three or more groups. A $p<0.05$ was considered significant.

\section{Results}

The results indicated that no significant difference was observed among the three groups with regard to the Gal3, LCN2, RBP, and sdLDL levels ( $p>0.05$ ) (Table 1).

It was also revealed that body weight, waist circumference, systolic and diastolic blood pressure, and BMI showed a significant difference among the three groups. The post hoc analysis indicated that this difference was between H-PCOS and the other two groups. Meaningfully, median values for body weight, waist circumference, systolic and diastolic blood pressure, and BMI were higher in the H-PCOS group than in the other two groups: body weight (median: $76.5 \mathrm{~kg}$ ) ( $p<0.001)$, waist circumference (median: $82.5 \mathrm{~cm})(p=0.001)$, systolic blood pressure (median: 110) ( $p=0.002)$, diastolic blood pressure (median: 70) ( $p=0.008)$, and BMI (median: $28.75 \pm 2.84$ ) $(p<0.001)$ (Table 2).

An analysis of the cholesterol levels among the three groups indicated that cholesterol and LDL cholesterol levels showed a significant difference among the three groups. The post hoc analysis also revealed that both cholesterol and LDL cholesterol levels were higher in the $\mathrm{H}$-PCOS group compared to the other two groups (median: 175.50 and $105.43 \pm 18.69 ; p=0.001$ and 0.003 , respectively) (Table 3).

\section{Discussion}

The results indicated that serum Gal3, LCN2, RBP, and sdLDL levels were normal in the $\mathrm{IH}$ and $\mathrm{H}$-PCOS groups compared to the control group. To our knowledge, this is the first study in the literature evaluating all these four parameters in patients with $\mathrm{IH}$ and $\mathrm{H}$-PCOS.

Li et al. showed that Gal3 is elevated in obese rats and humans. The authors also found that Gal3 administration in vivo causes systemic insulin resistance both acutely and chronically and in vitro inhibits insulin receptor activity in adipocytes, muscle cells, and hepatocytes. The authors concluded that Gal3 may be an important cause of systemic and cellular insulin resistance [22]. Recent reports indicate that Gal3 is elevated in patients with heart failure and atherosclerosis and Gal3 has been approved by the Food and Drugs Administration (FDA) as a marker of heart failure. In their review article, Agnello et al. suggested that Gal3 is an important prognostic marker for acute coronary syndrome [23]. Similarly, Xie et al. reported that Gal3 is closely associated with autoimmunity in rheumatoid arthritis and also plays a key role in the aetiology of rheumatoid arthritis [24]. Venkatraman et al. also showed that Gal3 is a practical biomarker and a promising molecule in haemorrhagic stroke

Table 1. Comparison of Gal3, LCN2, RBP, and sdLDL levels among the groups

\begin{tabular}{|c|c|c|c|c|c|c|}
\hline Parameter & & Median & IQR & Min. & Max. & $P$-value ${ }^{1}$ \\
\hline \multirow{3}{*}{$\begin{array}{l}\text { Galectin-3 } \\
{[\mathrm{pg} / \mathrm{ml}]}\end{array}$} & $\mathrm{H}-\mathrm{PCOS}$ & 742.245 & $414.865-1427.927$ & 201.291 & 2200.519 & \multirow[t]{3}{*}{0.643} \\
\hline & $\mathrm{IH}$ & 656.014 & 522.154-939.275 & 75.000 & 2229.769 & \\
\hline & Control & 850.035 & $469.011-2044.840$ & 75.000 & 2554.501 & \\
\hline \multirow{3}{*}{$\begin{array}{l}\text { Lipocalin } 2 \\
\text { [ng/l] }\end{array}$} & $\mathrm{H}-\mathrm{PCOS}$ & 1364.860 & $1050.000-2108.434$ & 200.000 & 3794.060 & \multirow[t]{3}{*}{0.212} \\
\hline & $\mathrm{IH}$ & 1065.470 & $974.183-1651.880$ & 200.000 & 4015.930 & \\
\hline & Control & 1122.720 & 200.000-1497.150 & 200.000 & 4034.070 & \\
\hline \multirow{3}{*}{$\begin{array}{l}\text { RBP } \\
{[\mathrm{ng} / \mathrm{ml}]}\end{array}$} & H-PCOS & 182.39 & $139.99-264.36$ & 25.00 & 493.49 & \multirow[t]{3}{*}{0.232} \\
\hline & $\mathrm{IH}$ & 169.96 & $116.78-221.96$ & 25.00 & 475.25 & \\
\hline & Control & 195.50 & $144.33-444.16$ & 25.00 & 503.91 & \\
\hline \multirow{3}{*}{$\begin{array}{l}\text { sdLDL } \\
{[\mathrm{mmol} / \mathrm{l}]}\end{array}$} & $\mathrm{H}-\mathrm{PCOS}$ & 1.81 & $1.20-3.61$ & 0.30 & 6.63 & \multirow[t]{3}{*}{0.874} \\
\hline & $\mathrm{IH}$ & 1.70 & $1.16-3.49$ & 0.30 & 6.12 & \\
\hline & Control & 1.64 & $1.35-4.06$ & 0.30 & 6.59 & \\
\hline
\end{tabular}

${ }^{1}$ Kruskal-Wallis Test, IH-idiopathic hirsutism, H-PCOS-PCOS with hirsutism, RBP-retinal binding protein, sdLDL-small, dense low-density lipoprotein. 
Table 2. Comparison of age, height, weight, waist circumference, blood pressure, and BMI among the groups

\begin{tabular}{|c|c|c|c|c|c|c|}
\hline Parameter & & Median & IQR & Min. & Max. & $P$-value \\
\hline \multirow[t]{3}{*}{ Age [years] } & H-PCOS & 23.00 & $19.00-25.00$ & 17.00 & 33.00 & \multirow[t]{3}{*}{0.813} \\
\hline & $\mathrm{IH}$ & 22.00 & $20.00-25.00$ & 16.00 & 31.00 & \\
\hline & Control & 21.50 & $19.00-23.00$ & 17.00 & 33.00 & \\
\hline \multirow[t]{3}{*}{ Height [cm] } & H-PCOS & 162.00 & $158.00-165.00$ & 152.00 & 173.00 & \multirow[t]{3}{*}{0.551} \\
\hline & $\mathrm{IH}$ & 161.00 & $159.00-164.00$ & 155.00 & 171.00 & \\
\hline & Control & 161.00 & $158.00-163.00$ & 155.00 & 172.00 & \\
\hline \multirow[t]{3}{*}{ Weight [kg] } & H-PCOS & 76.50 & $68.00-82.00$ & 60.00 & 97.00 & \multirow[t]{3}{*}{$<0.001$} \\
\hline & $\mathrm{IH}$ & 59.50 & $56.00-63.00$ & 49.00 & 83.00 & \\
\hline & Control & 61.00 & $57.00-63.00$ & 50.00 & 84.00 & \\
\hline \multirow{3}{*}{$\begin{array}{l}\text { Waist circumference } \\
{[\mathrm{cm}]}\end{array}$} & H-PCOS & 82.50 & $76.00-94.00$ & 60.00 & 105.00 & \multirow[t]{3}{*}{0.001} \\
\hline & $\mathrm{IH}$ & 73.00 & $68.00-81.00$ & 62.00 & 95.00 & \\
\hline & Control & 74.50 & $70.00-80.00$ & 65.00 & 101.00 & \\
\hline \multirow{3}{*}{$\begin{array}{l}\text { Systolic blood } \\
\text { pressure [mm Hg] }\end{array}$} & H-PCOS & 110.00 & $100.00-120.00$ & 90.00 & 130.00 & \multirow[t]{3}{*}{0.002} \\
\hline & $\mathrm{IH}$ & 100.00 & $90.00-110.00$ & 60.00 & 120.00 & \\
\hline & Control & 100.00 & $100.00-100.00$ & 90.00 & 120.00 & \\
\hline \multirow{3}{*}{$\begin{array}{l}\text { Diastolic blood } \\
\text { pressure [mm Hg] }\end{array}$} & H-PCOS & 70.00 & $70.00-80.00$ & 60.00 & 80.00 & \multirow[t]{3}{*}{0.008} \\
\hline & $\mathrm{IH}$ & 60.00 & $60.00-70.00$ & 50.00 & 90.00 & \\
\hline & Control & 65.00 & $60.00-70.00$ & 50.00 & 80.00 & \\
\hline \multirow[t]{3}{*}{$\mathrm{BMI}\left[\mathrm{kg} / \mathrm{m}^{2}\right]$} & H-PCOS & \multicolumn{2}{|c|}{$28.75 \pm 2.84$} & 23.40 & 33.90 & \multirow[t]{3}{*}{$<0.001$} \\
\hline & $\mathrm{IH}$ & \multicolumn{2}{|c|}{$23.27 \pm 3.06$} & 18.10 & 30.10 & \\
\hline & Control & \multicolumn{2}{|c|}{$23.55 \pm 2.82$} & 17.30 & 28.80 & \\
\hline
\end{tabular}

${ }^{1}$ Kruskal Wallis Test, ${ }^{2}$ ANOVA Test results were expressed as mean \pm SD instead of median and IQR, IH -idiopathic hirsutism, H-PCOS - PCOS with hirsutism, $B M I$ - body mass index.

Table 3. Comparison of cholesterol levels among the three groups

\begin{tabular}{|c|c|c|c|c|c|c|}
\hline Parameter & & Median & IQR & Min. & Max. & $P$-value ${ }^{1}$ \\
\hline \multirow[t]{3}{*}{ HDL cholesterol } & $\mathrm{H}-\mathrm{PCOS}$ & 44.50 & $41.00-50.00$ & 31.00 & 79.00 & 0.260 \\
\hline & $\mathrm{IH}$ & 44.50 & $40.00-49.00$ & 27.00 & 54.00 & \\
\hline & Control & 47.00 & $43.00-52.00$ & 29.00 & 66.00 & \\
\hline \multirow[t]{3}{*}{ cholesterol } & $\mathrm{H}-\mathrm{PCOS}$ & 175.50 & $160.00-185.00$ & 140.00 & 252.00 & 0.001 \\
\hline & $\mathrm{IH}$ & 160.00 & $139.00-167.00$ & 112.00 & 271.00 & \\
\hline & Control & 154.00 & 133.00-170.00 & 118.00 & 190.00 & \\
\hline \multirow[t]{3}{*}{ LDL cholesterol } & H-PCOS & \multicolumn{2}{|c|}{$105.43 \pm 18.69$} & 79.00 & 150.00 & $0.003^{2}$ \\
\hline & $\mathrm{IH}$ & \multicolumn{2}{|c|}{$97.57 \pm 30.72$} & 55.00 & 214.00 & \\
\hline & Control & \multicolumn{2}{|c|}{$84.43 \pm 18.02$} & 55.00 & 112.00 & \\
\hline
\end{tabular}

${ }^{1}$ Kruskal Wallis Test, ${ }^{2}$ ANOVA Test results were expressed as mean \pm SD instead of median and IQR, IH - idiopathic hirsutism, H-PCOS - PCOS with hirsutism.

and cerebrovascular diseases [25]. In addition, Feng et al. indicated that Gal3 is elevated in acute exacerbation of chronic obstructive pulmonary disease (COPD) and that Gal3 is a valuable biomarker for this disease [26]. On the other hand, Saccon et al. reported that galectins play a key role in renal haemostasis and several other renal diseases and also suggested that Gal3 is an important control point in acute and chronic inflammation.
The authors also noted that Gal3 plays a pivotal role in wound repair although it causes fibrosis in various organs and concluded that Gal3 can be a useful marker in renal diseases [27]. Yilmaz et al. also reported that Gal3 was elevated in patients with PCOS and showed that this elevation was accompanied by insulin resistance and hyperandrogenemia. The authors concluded that Gal3 plays a key role in the aetiology of PCOS [12]. In our study, Gal3 
levels showed no significant difference between the $\mathrm{H}$ PCOS and IH groups and the control group, which suggests that Gal3 cannot be a useful marker in hirsutism and PCOS. Moreover, the finding that Gal3 levels were found to be elevated in the PCOS patients reported in previous studies and were found to be normal in our PCOS patients could be attributed to the fact that our PCOS patients also co-presented with hirsutism. To our knowledge, our study is the first report in the literature to investigate the Gal3 level in patients with $\mathrm{IH}$.

Jiao et al. reported that elevated serum levels of RBP were associated with an increased risk of breast cancer. Moreover, the authors showed that this elevation was independent of BMI and serum lipids and was not associated with the type of breast cancer [28]. Yang et al. [29] and Graham et al. [30] reported that the RBP synthesized by the adipocytes may result in metabolic diseases including hepatic diseases. Dong et al. also noted that elevated RBP levels pose an important risk factor for coronary artery disease and for the formation of plaques in the carotid artery. The authors suggested that early detection and regulation of RBP elevation is of crucial importance [31]. Kokkinos et al. found that serum RBP levels were elevated in patients with hyperthyroidism but were normalized after the treatment. The authors concluded that RBP is a key marker in thyroid dysfunction [32]. Jeon et al. also found that RBP levels were significantly elevated in patients with PCOS compared to control subjects [33]. Weiping et al. reported that an elevated RBP level was associated with insulin resistance in PCOS patients [34]. Mellati et al. also found that RBP levels were elevated in PCOS patients and this elevation was associated with androgen hormones but not with insulin resistance [35]. Although RBP has been reported to be elevated in PCOS and some other diseases in previous studies, we found normal RBP levels in patients with $\mathrm{IH}$ and PCOS. Therefore, we believe that RBP cannot be a useful marker in $\mathrm{IH}$ and PCOS patients. Moreover, the finding that the patients in the H-PCOS group had normal RBP levels despite having higher values for waist circumference and body weight suggests that RBP is not associated with body fat percentage.

Shen et al. evaluated 115 patients with severe brain injury and 115 control subjects and reported that LCN2 levels were significantly higher in patients with acute brain injuries and thus LCN2 could be an important prognostic marker in such patients [36]. Flo et al. compared LCN2-deficient rats with control rats and reported that LCN2 provides protection against systemic E. coli infections [37, 38]. Similarly, Berger et al. found that LCN2 provides protection against systemic E. coli sepsis [37, 38]. Tsuda et al. reported that the gene expression levels of LCN2 were significantly decreased in lung hypoplasia in rats and in congenital diaphragmatic hernias in humans and thus LCN2 is a useful biomarker in the diagnosis of such diseases [39]. Wu et al. suggested that LCN2 is a valuable marker in the early diagnosis and treatment of cardiovascular diseases [40]. Choi et al. found that there was no significant difference in LCN2 levels between obese and non-obese individuals and that the LCN2 levels did not change following exercise training with weight loss [41]. However, Yilmaz et al. found that although LCN2 was elevated in patients with PCOS, it was not associated with insulin resistance and obesity but was significantly associated with serum androgen levels [12]. Similarly, Ozkaya et al. found elevated LCN2 levels in women with PCOS [42]. Conversely, Koiou et al. found normal LCN2 levels in PCOS patients [43]. Panidis et al. found no association between serum LCN2 levels and PCOS but suggested that LCN2 could be a valuable marker for insulin resistance in obese patients [19]. Gencer et al. reported that LCN2 was decreased in PCOS patients and there was no relationship between LCN2 and cardiovascular disease [44]. Although LCN2 has been reported as a novel biomarker in previous studies, we found normal LCN2 levels in our patients and obtained similar results with Koiou et al. and Panidis et al. To the best of our knowledge, our study is the first report in the literature to investigate LCN2 levels in patients with $\mathrm{IH}$.

Low-density lipoprotein (LDL) consists of several subgroups of particles with different sizes and densities, including large buoyant (lb), intermediate, and small dense (sd) LDLs. In recent reports, sdLDL has been shown to be more atherogenic than the other subgroups and total LDL and to be an important marker in the development of cardiovascular diseases [45, 46]. Kulanuwat et al. evaluated a Thai population and reported that sdLDL increased with obesity and also led to an increased risk of obesity and atherosclerosis [47]. However, Phelan et al. found no significant difference in SdLDL levels between women with PCOS and control subjects [48]. Kocer et al. found increased sdLDL levels and decreased highdensity lipoprotein (HDL) levels in patients with PCOS. The authors also noted that the patients had an excess risk of cardiovascular disease and metabolic syndrome and these risks could be eliminated by metformin treatment [49]. Kim et al. also found increased sdLDL levels in patients with PCOS and suggested that dyslipidemia could be the primary indicator of metabolic syndrome in patients with PCOS [50]. In our study, serum sdLDL levels showed no significant difference between the patients with $\mathrm{IH}$ and $\mathrm{H}-\mathrm{PCOS}$ and the control subjects, similar to the findings presented by Phelan et al. Meaningfully, this finding suggests that sdLDL cannot be a useful marker in patients with hirsutism. Our study was limited since it had a small number of patients and was a single-centre study.

Our results also indicated that the patients with PCOS had normal Gal3, LCN2, RBP, and sdLDL levels although they had greater body weight and waist circumference compared to the other groups, which suggests that these four parameters did not correlate with body 
weight. The literature reviews demonstrate that although the relationship between adipose tissue and its metabolism and PCOS has been extensively investigated, there have been very few studies reporting on this relationship in patients with $\mathrm{IH}$. In the present study, we aimed to evaluate this relationship in patients with $\mathrm{IH}$ by analysing four key parameters.

\section{Conclusions}

No significant correlation was found between the adipokines that play a role in the aetiology of numerous diseases and some mediators of the lipid metabolism and hirsutism. Nevertheless, further multi-centre studies are needed to present more substantial findings related to this relationship.

\section{Acknowledgments}

This work was supported by the Research Fund of the Yuzuncu Yil University.

\section{Conflict of interest}

The authors declare no conflict of interest.

\section{References}

1. Pate C. The story plot of living the embarrassment of hirsutism. Arch Psychiatr Nurs 2013; 27: 156-7.

2. Hohl A, Ronsoni MF, Oliveira M. Hirsutism: diagnosis and treatment. Arq Bras Endocrinol Metabol 2014; 58: 97-107.

3. Azziz R, Carmina E, Sawaya ME. Idiopathic hirsutism. Endocr Rev 2000; 21: 347-62.

4. Abbott DH, Dumesic DA, Franks S. Developmental origin of polycystic ovary syndrome - a hypothesis. J Endocrinol 2002; 174: 1-5.

5. Sortino MA, Salomone S, Carruba MO, et al. Polycystic ovary syndrome: insights into the therapeutic approach with inositols. Front Pharmacol 2017; 8: 341.

6. De Leo V, Musacchio MC, Cappelli V, et al. Genetic, hormonal and metabolic aspects of PCOS: an update. Reprod Biol Endocrinol 2016; 14: 38.

7. Rosenfield RL. The diagnosis of polycystic ovary syndrome in adolescents. Pediatrics 2015; 136: 1154-65.

8. Lau DC, Dhillon B, Yan H, et al. Adipokines: molecular links between obesity and atheroslcerosis. Am J Physiol Heart Circ Physiol 2005; 288: H2031-41.

9. Ferroni P, Basili S, Falco A, et al. Inflammation, insulin resistance, and obesity. Curr Atheroscler Rep 2004; 6: 424-31.

10. Fantuzzi G. Adipose tissue, adipokines, and inflammation. J Allergy Clin Immunol 2005; 115: 911-9.

11. Henderson NC, Sethi T. The regulation of inflammation by galectin-3. Immunol Rev 2009; 230: 160-71.

12. Yilmaz H, Celik HT, Ozdemir O, et al. Serum galectin-3 levels in women with PCOS. J Endocrinol Invest 2014; 37: 181-7.

13. Arar C, Gaudin JC, Capron LC, et al. Galectin-3 gene (LGALS3) expression in experimental atherosclerosis and cultured smooth muscle cells. FEBS Lett 1998; 430: 307-11.

14. Benoldi D, Manfredi G, Pezzarossa E, et al. Retinol binding protein in normal human skin and in cutaneous disorders. Br J Dermatol 1981; 105: 659-65.
15. Tan BK, Chen J, Lehnert $H$, et al. Raised serum, adipocyte, and adipose tissue retinol-binding protein 4 in overweight women with polycystic ovary syndrome: effects of gonadal and adrenal steroids. J Clin Endocrinol Metab 2007; 92: 2764-72.

16. Aigner E, Bachofner N, Klein K, et al. Retinol-binding protein 4 in polycystic ovary syndrome: association with steroid hormones and response to pioglitazone treatment. J Clin Endocrinol Metab 2009; 94: 1229-35.

17. Triebel S, Blaser J, Reinke H, et al. A 25 kDa alpha 2-microglobulin-related protein is a component of the $125 \mathrm{kDa}$ form of human gelatinase. FEBS Lett 1992; 314: 386-8.

18. Kjeldsen L, Johnsen AH, Sengeløv H, et al. Isolation and primary structure of NGAL, a novel protein associated with human neutrophil gelatinase. J Biol Chem 1993; 268: 10425-32.

19. Panidis D, Tziomalos K, Koiou E, et al. The effects of obesity and polycystic ovary syndrome on serum lipocalin-2 levels: a cross-sectional study. Reprod Biol Endocrinol 2010; 8: 151.

20. Kim JJ, Chae SJ, Choi YM, et al. Atherogenic changes in lowdensity lipoprotein particle profiles were not observed in non-obese women with polycystic ovary syndrome. Hum Reprod 2013; 28: 1354-60.

21. Rotterdam ESHRE/ASRM-Sponsored PCOS consensus workshop group. Revised 2003 consensus on diagnostic criteria and long-term health risks related to polycystic ovary syndrome (PCOS). Hum Reprod 2004;19: 41-7.

22. Li P, Liu S, Lu M, et al. Hematopoietic-derived galectin-3 causes cellular and systemic insulin resistance. Cell 2016; 167: 973-84. e12.

23. Agnello L, Bivona G, Lo Sasso B, et al. Galectin-3 in acute coronary syndrome. Clin Biochem 2017; 50: 797-803.

24. Xie Q, Ni M, Wang SC. Galectin-3, a potential therapeutic target for rheumatoid arthritis? Scand I Immunol 2017.12631.

25. Venkatraman A, Hardas S, Patel N, et al. Galectin-3: an emerging biomarker in stroke and cerebrovascular diseases. Eur J Neurol 2017; 25: 238-46.

26. Feng $W, W u X$, Li S, et al. Association of serum galectin-3 with the acute exacerbation of chronic obstructive pulmonary disease. Med Sci Monit 2017; 23: 4612-8.

27. Saccon F, Gatto M, Ghirardello A, et al. Role of galectin-3 in autoimmune and non-autoimmune nephropathies. Autoimmun Rev 2017; 16: 34-47.

28. Jiao C, Cui L, Ma A, et al. Elevated serum levels of retinolbinding protein 4 are associated with breast cancer risk: a case-control study. PloS One 2016; 11: e0167498.

29. Yang Q, Graham TE, Mody N, et al. Serum retinol binding protein 4 contributes to insulin resistance in obesity and type 2 diabetes. Nature 2005; 436: 356-62.

30. Graham TE, Yang Q, Bluher M, et al. Retinol-binding protein 4 and insulin resistance in lean, obese, and diabetic subjects. N Engl J Med 2006; 354: 2552-63.

31. Dong H, Li X, Tang Y. Serum retinol-binding protein-4 level is a high risk factor for coronary heart disease in Chinese. Clin Lab 2015; 61: 1675-8.

32. Kokkinos S, Papazoglou D, Zisimopoulos A, et al. Retinol binding protein-4 and adiponectin levels in thyroid overt and subclinical dysfunction. Exp Clin Endocrinol Diabetes 2016; 124: 87-92.

33. Jeon YE, Lee KE, Jung JA, et al. Kisspeptin, leptin, and retinolbinding protein 4 in women with polycystic ovary syndrome. Gynecol Obstet Invest 2013; 75: 268-74.

34. Weiping L, Qingfeng C, Shikun M, et al. Elevated serum RBP4 is associated with insulin resistance in women with polycystic ovary syndrome. Endocrine 2006; 30: 283-7. 
35. Mellati AA, Sharifi F, Sajadinejad M, et al. The relationship between retinol-binding protein 4 levels, insulin resistance, androgen hormones and polycystic ovary syndrome. Scand J Clin Lab Invest 2012; 72: 39-44.

36. Shen LJ, Zhou J, Guo M, et al. Serum lipocalin-2 concentrations and mortality of severe traumatic brain injury. Clin Chim Acta 2017; 474: 130-5.

37. Flo TH, Smith KD, Sato S, et al. Lipocalin 2 mediates an innate immune response to bacterial infection by sequestrating iron. Nature 2004; 432: 917-21.

38. Berger T, Togawa A, Duncan GS, et al. Lipocalin 2-deficient mice exhibit increased sensitivity to Escherichia coli infection but not to ischemia-reperfusion injury. Proc Natl Acad Sci USA 2006; 103: 1834-9.

39. Tsuda H, Kotani T, Nakano T, et al. Lipocalin 2 as a new biomarker for fetal lung hypoplasia in congenital diaphragmatic hernia. Clin Chim Acta 2016; 462: 71-6.

40. Wu G, Li H, Fang Q, et al. Elevated circulating lipocalin-2 levels independently predict incident cardiovascular events in men in a population-based cohort. Arterioscler Thromb Vasc Biol 2014; 34: 2457-64.

41. Choi KM, Kim TN, Yoo HJ, et al. Effect of exercise training on A-FABP, lipocalin-2 and RBP4 levels in obese women. Clin Endocrinol (Oxf) 2009; 70: 569-74.

42. Ozkaya M, Engin-Ustun Y, Ustun Y. Serum lipocalin-2 as an insulin resistance marker in patients with polycystic ovary syndrome. J Endocrinol Invest 2011; 34: 97-100.

43. Koiou E, Tziomalos K, Katsikis I, et al. Weight loss significantly reduces serum lipocalin-2 levels in overweight and obese women with polycystic ovary syndrome. Gynecol Endocrinol 2012; 28: 20-4.

44. Gencer M, Gazi E, Hacıvelioğlu S, et al. The relationship between subclinical cardiovascular disease and lipocalin-2 levels in women with PCOS. Eur J Obstet Gynecol Reprod Biol 2014; 181: 99-103.

45. Ivanova EA, Myasoedova VA, Melnichenko AA, et al. Small dense low-density lipoprotein as biomarker for atherosclerotic diseases. Oxid Med Cell Longev 2017; 2017: 1273042.

46. Gerber PA, Nikolic D, Rizzo M. Small, dense LDL: an update. Curr Opin Cardiol 2017; 32: 454-9.

47. Kulanuwat S, Tungtrongchitr R, Billington D, et al. Prevalence of plasma small dense LDL is increased in obesity in a Thai population. Lipids Health Dis 2015; 14: 30.

48. Phelan N, O'Connor A, Kyaw-Tun T, et al. Lipoprotein subclass patterns in women with polycystic ovary syndrome (PCOS) compared with equally insulin-resistant women without PCOS. J Clin Endocrinol Metab 2010; 95: 3933-9.

49. Kocer D, Bayram F, Diri H. The effects of metformin on endothelial dysfunction, lipid metabolism and oxidative stress in women with polycystic ovary syndrome. Gynecol Endocrinol 2014; 30: 367-71.

50. Kim JJ, Choi YM. Dyslipidemia in women with polycystic ovary syndrome. Obstet Gynecol Sci 2013; 56: 137-42. 